\title{
Internet Gambling and Social Responsibility: An Exploratory Study
}

MICHAEL SMEATON ${ }^{1}$ and MARK GRIFFITHS ${ }^{2}$

Au:

Pls. provide academic degrees for authors.

\begin{abstract}
Within the last few years, we have seen a substantial increase in the amount of gambling opportunities offered on the Internet. Many concerns have been raised about the activity, including the potential for excessive gambling, and the lack of safeguards for vulnerable populations such as adolescents and problem gamblers. Given these concerns, a representative selection of 30 U.K.-owned Internet gambling sites were studied to examine what safeguards were in place to encourage social responsibility of Internet gamblers. It was found that very few sites were engaged in socially responsible practice and that much more could be done to protect vulnerable groups. The findings from this study were then used to compile a list of recommendations for good practice among Internet gambling operators.
\end{abstract}

\section{INTRODUCTION}

W THIN THE LAST FEW YEARS, we have seen a substantial increase in the amount of gambling opportunities offered on the Internet. This has been noted by many observers and organizations. ${ }^{1-4}$ With the number of sites rising all the time, there is no doubt that this market will grow further over the next few years, increasing gambling opportunities for the customer and possibly attracting new customers to this industry. The dilemma for this new market is how to ensure the future of Internet gambling is regulated, controlled, and socially responsible. Whilst the Gaming Board for Great Britain is obviously concerned with the complexities of legislation, regulation, and taxing of the Internet gaming industries, the authors' concerns are directed towards the social responsibility of these sites.

The emergence of Internet gambling means that a new and very different delivery platform for gambling has come into the mainstream gambling industry. The 24-h accessibility and availability of gambling opportunities can be provided in the comfort of a person's own home or workplace. Whilst having benefits for the gaming industry and the customer, such a situation could hold certain risks for both the industry, and the customer who may be unable to control their gambling. A number of U.K.-based organizations (such as GamCarethe National Association on Gambling) have done a lot of work in the past with the gambling industry. Their prime aim is to help the gaming industry to develop a socially responsible approach towards its customers, in order to protect the vulnerable (e.g., adolescents, problem gamblers, those under the influence of alcohol/drugs).

The authors would like to see the gaming industry continue the socially responsible stance that many have adopted in the past. This is especially relevant considering the new risks and dilemmas

${ }^{1}$ GamCare, London, United Kingdom

${ }^{2}$ Psychology Division, Nottingham Trent University, Nottingham, United Kingdom. 
that will be faced as Internet gaming grows. Although not an exhaustive list, the following areas of concern differentiate Internet gambling from the traditional gambling mediums.

- Access and availability to gambling $24 \mathrm{~h}$ a day, 365 days a year, has the potential to lead to excessive gambling behavior. ${ }^{1,2}$

- Some accepted forms of payment at Internet gambling sites mean that they are unable to immediately determine whether the customer is within the legal age, unless stringent checks are made on every new customer.

- Allowing access to gambling into homes could lead to younger children being exposed to gambling and gives control to the adults to let their children participate in gambling. ${ }^{2}$

- The perception of the value of money decreases when playing with electronic cash, as it has the ability to suspend judgment in much the same way as chips used in casinos.1,3 This could increase the risk of excessive gambling and accumulation of debts.

- There will be no control over those who may be gambling whilst drunk or on drugs. ${ }^{1}$

- Any possible intervention to help someone who may have a gambling problem will be extremely limited, due to the lack of personal contact with that customer.

- Gambling on the Internet could shift gambling from a social activity into a more asocial one, which could lead to a greater risk of problem gambling. ${ }^{1}$

- The customer may be at risk from offshore, unregulated, and disreputable Internet gaming sites, unless able to differentiate between such sites and socially responsible, regulated and reputable ones. ${ }^{1}$

With these concerns in mind, a representative selection of U.K.-owned Internet gambling sites were studied to examine what safeguards were in place to encourage the social responsibility of Internet gamblers. The findings from this study are then used to compile a list of recommendations for good practice.

\section{MATERIALS AND METHODS}

An exploratory study examined 30 Internet gambling sites (operated by 27 different gaming companies) that had a direct link with the U.K. (see Appendix 1 for a complete list of sites studied and their website address). Each site was visited and then examined in further detail by asking a number of questions. More specifically, did the internet gambling site:

- Do an initial age check of the player?

- Do an age verification check of the player?

- Do a credit check on the player?

- Have credit limits for the players?

- Make reference to controlled gambling?

- Make link to helping organisations and/or selfhelp groups?

- Show evidence of social responsibility?

- Have a facility for gamblers who wanted to exclude themselves?

- Have a facility to instantly exit during gambling?

- Have a built-in pause/confirmation facility?

- Have a "practice mode" facility?

- Give encouragement to continue gambling?

- Give players easy access to their account balance?

- Have a wide range of payment methods (e.g., credit/debit cards, personal checks, banker's check/draft, wire transfer)

\section{RESULTS}

The findings from the study varied a great deal. There were some very positive findings from sites that clearly displayed responsible practice, whilst other sites practices were a cause for concern. Each of these main areas of concern is briefly described. Each section also contains some preliminary discussion.

\section{Check on age}

Of the 30 sites visited, half of them $(n=15)$ did not carry any age warning at all. Twelve of the sites had age restrictions (all 18 years of age or older apart from a couple in which the player had to be 16 years or older), although on one of these the age restriction was buried away in the small print and was not easy to find. Three of the sites did not explicitly have age restrictions, although they did point out in their terms and conditions that proof of age could be sought if they so wished. Overall, about half the sites make it clear that underage gambling is prohibited. This is an area that many sites can improve on.

\section{Age check verification on registration}

Of the 30 sites visited, 11 had no age verification check when registering at the site. However, 
five of these sites required credit card details to register, which means the number of adolescents who may have tried to access these sites is probably quite low unless they were fraudulently using the cards. Seventeen sites had some kind of age verification check, although these were split between those where the person registering just had to tick a box to say they were 18 years of age or older $(n=10)$ and those where the person simply had to enter their date of birth. Unfortunately, both of these types of age verification are open to abuse if the person lies about their age. There were a further two sites where it was hard to establish whether there was any kind of age verification. Overall, it was clear that there was very little in the way of age verification from any site. The current system is open to abuse, and there is little to stop a determined adolescent who wants to gamble.

\section{Credit checks}

Of the 30 sites visited, there were only four sites where there was any evidence of a credit check being done on the gambler and only one site that said they did not run credit checks. In the remaining sites, it was unclear whether credit checks were run or not. It would appear that running a credit check is not standard practice. Such a practice might exclude vulnerable groups from gambling (adolescents and problem gamblers).

\section{Credit limits}

There was a large range of credit limits across the 30 sites visited. The authors located only three sites that had no information about either minimum or maximum bet size. The minimum bet size found was $£ 1$, whereas the maximum located (of the sites that set an upper limit) was $£ 20,000$. Many of the sites typically had £10-25 minimum bets, and $£ 250-1000$ maximum bets. Obviously, the larger the credit limit, the more chance that a gambler can run up debts that they cannot afford. Given the seemingly lax approach to both age verification and credit checks, this is an area that requires further monitoring and guidelines.

\section{Reference to controlled gambling}

Of the 30 sites visited, 23 had no reference whatsoever to controlled gambling. Of the seven sites that did make some reference, some of these were very mild references (such as a single statement with a "risk warning"). Overall, raising awareness about controlled gambling appears to be very low on priority for Internet gambling operators.

\section{Reference to a gambling help group}

Of the 30 sites visited, 26 had no reference to either a gambling telephone helpline, a gambling help organization, or a problem gambling self-help group. Of the four sites that did make a reference, only two had an active hyperlink. Two sites displayed the GamCare logo, but there was no link to the GamCare homepage. Given the potential for problems with Internet gambling, it was perhaps surprising that hardly any site provided links to helping agencies. This is one area where there is large scope for improvement.

\section{Evidence of social responsibility}

Of the 30 sites visited, two-thirds of sites $(n=20)$ showed no evidence of any social responsibility. The remaining 10 sites were varied in the level of social responsibility displayed, although the majority of social responsibility statements on these sites was somewhat minimal. Like references to controlled gambling, social responsibility appears to be very low on priority for Internet gambling operators.

\section{Self-exclusion}

Of the 30 sites visited, there was no evidence from 29 sites that gamblers could exclude themselves from the site. One site had the option to lower the site's own credit limit, but there was nothing explicit about the capacity to self-exclude. This again is an area where there is almost unanimous scope for improvement.

\section{Instant exit}

Of the 30 sites visited, just over one-third $(n=11)$ had clear instant exit facilities for gamblers. However, it was unclear how many of the remaining 19 sites had instant exit for gamblers. The fact that the authors were unable to locate such a facility suggests that it might be difficult for gamblers to do so even if there is one.

\section{Built-in pause/confirmation}

Of the 30 sites visited, a third $(n=10)$ had some kind of pause or "confirmation of bet" facility (although one of these could only be done on the telephone rather than online). Five sites had no such facility, with the remaining 15 sites unknown, as 
the authors could not find any evidence either way. However, it is our contention that these sites are less likely to have these features, particularly if the authors could not locate them on our visits. This is an area that Internet gambling operators must address (and is returned to again below).

\section{Practice modes}

Of the 30 sites visited, under a half had no practice modes $(n=13)$. Three sites had explicit practice modes, with a further seven sites where the gambler can enter or browse as a "guest." Three other sites had "demo" versions that could be played or practiced upon, and one site offered a free game to any gambler. In three sites, the authors were unable to ascertain whether there was a practice mode or not. The results suggest that adolescents have the potential to access almost any site that has a practice or "demo" facility. This is a potentially worrying situation and is expanded on in the next section.

\section{Encouragement to gamble}

Of the 30 sites visited, the authors identified evidence at only two sites that encouraged continued gambling (e.g., one site placed winnings directly back into the betting account and gamblers are told to keep their money in there for further bets). However, there were only five sites where the authors could absolutely say there was no encouragement to continue gambling. Many sites engaged in practices that border on encouragement (e.g., sites that e-mailed the gambler after they had lost at the site and invited them to come back and "have another go" $^{\prime \prime}$. Other sites offered free bets, free memberships to casinos, and being entered into prize draws after gambling a certain number of times). This is one area that needs to be monitored carefully as there is a very fine line between legitimate marketing practices and exploitation.

\section{Access to account balance}

Of the 30 sites visited, two-thirds of sites $(n=20)$ provided easy access to account balances (although one of these was by telephone only). In the remaining cases, it was unclear how to even access the balance. Overall, the sites did well on this particular criterion although there is clearly room for improvement from some sites.

\section{Methods of payment}

Almost all of the 30 sites visited accepted either credit cards $(n=26)$ and/or debit cards $(n=23)$. Other forms of acceptable payment included personal checks $(n=13)$, banker's check $(n=6)$, banker's draft $(n=6)$, wire transfer $(n=3)$, and other types of payment $(n=3$; postal order, electronic check). Given that only credit cards are restricted to adults, it is a potential worry that other forms of payment that adolescents have access to are acceptable forms of payment.

\section{CONCLUSIONS}

Each of the areas covered will now be discussed. Not only will "good practice" and "bad practice" be highlighted, but recommendations will also be given.

\section{Check on age}

Age checks should be compulsory for all new accounts. This procedure should be clearly displayed within the registration page and within the rules and regulations section, so as to deter attempted under-age playing. There should be a clear sign on the home page that gambling on the site is permitted to over 18's only, unless another age limit applies.

Good practice. Very few of the sites visited demonstrated good practice. Not only should age checks be carried out on as many new players as possible, but this practice should be clearly displayed in rules and registration section. In the U.K., the technology is available to carry out electoral roll checks on new customers (i.e., if players appear on the adult electoral roll register they must be by definition at least 18 years old). Although this is not a foolproof system, it is better than no action at all.

Bad practice. There were too many sites whose approach to age checking is a cause for concern. A lot of sites show no evidence of validating age, and merely rely on ticking a box to verify being over 18 years of age.

\section{Age check registration}

Any customer wishing to register should have their age verified before their application is accepted. This procedure should be clearly displayed 
in the registration page, thus ensuring that no one under age is able to access the member's pages on the site, and have the opportunity to set up an account.

Good practice. There was no really a good example of clear procedures of age checking being displayed on the registration page. The nearest examples found were pages that required the individual to enter their date of birth (at least a possible opportunity to identify those underage).

Bad practice. As mentioned above, there were too many sites that just required a box to be ticked verifying age in the registration page. There were no messages saying why the player must be over 18 years or whether age checks are carried. It appears pointless to ask a customer to verify their age, without checking the information submitted.

\section{Methods of payment}

If age checks cannot be carried out on all new customers, then methods of payment should be restricted to credit cards only. All other forms of payment such as debit cards, personal and bankers' checks, wire transfers, and postal orders are available to individuals under the age of 18 years, and therefore could lead to underage online gambling.

Good practice. There were only a couple of sites that accepted credit cards only. This seems to be the only way of ensuring that the player is over 18 years (i.e., the age required to legally have a credit card). Any other forms of payment should be accompanied with an age verification system.

Bad practice. There were quite a few sites that accept payment methods such as personal checks and bankers' drafts. These are methods that are available to those under 18 years and do not appear to be accompanied by any age verification procedure. One site even accepted postal orders, which can be used by anyone with a post office account, no matter what age.

\section{Credit limits}

These should be clearly displayed within the rules and regulations and preferably within the registration page. Each customer should have the option to set their own credit limit, so that they may have some form of control over their gambling expenditure.
Good practice. The authors are aware that at least one online gaming site is looking into implementing a system where customers can select their own credit limit over a period of that player's choice. Whilst the authors endorse such a system, it is important that these limits should be easy to set up, but harder to change. A self-imposed credit limit may be used by an individual who is concerned about controlling their gambling, but if it is as easy to increase as it is to decrease, its use will be ineffective.

Bad practice. A number of sites did not have any credit limits or an option to set up one. Whilst some of these sites may actually have a credit limit, this information should be displayed clearly.

\section{Reference to controlled gambling}

There should be a reference to the need to keep gambling under control (i.e., a "risks of the game" message) similar to those displayed on most spread betting sites. This message should be displayed where it will be read, such as on the homepage or the registration page. This message should also be accompanied by a link to the website of the preferred social responsibility partner (e.g., GamCare) and a statement as to why the company supports them. A notice as to where a gambler who is concerned with their (or someone else's) gambling can get help should appear at least twice on an online gambling website. One of these should be on the homepage and one on the page where a customer can transfer money into their gambling account. This message could also include a link to any relevant website (e.g., GamCare).

Good practice. Some sites already carry the logos of gambling help organizations, with either a link to that website or a link to a message about responsible gambling together with a helpline number. Such a notice about controlled gambling and reference to a helpline should be prominently located on the homepage of the site, and the account details page. (This is because an individual transferring money is more likely to think about their gambling.) Warning messages are routinely placed on spread betting sites, which make the customer aware of the risks involved in such an activity.

Bad practice. The authors would obviously like to see every site with a message of responsible gambling and reference to where they can get help or advice. Not only will this benefit any gambler 
who may desire help, but it will also display a level of social responsibility by the company. As can be seen in the results section, only a small number of sites display these references. The majority still do not hold any information for help and advice for their customers.

\section{Self-exclusion}

There should be the option for customers to bar themselves from any online gambling site.

Good practice. Whilst the authors are not aware of any schemes currently in place online, where a person is able to bar themselves from a site, this is an option that the authors would like to see available for the customer. Any procedure for self-exclusion should be clearly stated in the rules and procedures page, with a minimum exclusion period of 6 months.

Bad practice. Any site that does not provide a self-exclusion service is openly encouraging further play. This is especially relevant for online gambling as companies are now able to keep track of player behavior online. This opportunity could be exploited detecting those customers that may be showing signs of excessive gambling and encouraging them to gamble further.

\section{Accreditation or standard bearer}

Although accreditation is something that cannot be directly implemented by an Internet gaming site, it would be good practice to have a standard that can be followed by all existing and emerging sites. Such sites that follow Government regulations, fair practice, and a socially responsible stance, should be accredited with an approval stamp, endorsed by the regulator. This will allow the customer to make an informed choice on the site they wish to use for their gambling activities. Such a recognized stamp of approval would be more likely to attract the customer to such a site.

Good practice. A good example of such practice is shown by the American Internet Gaming Commission. This is an independent portal that categorizes online sites on its website. It offers accreditation to online sites and will recommend these sites. It also lists non-accredited sites and will give cautions to sites it has concerns over. All this leads to the customer being able to make an informed choice on the sites it wishes to use. All ac- credited sites must follow a strict code of conduct, which includes protection to minors and other socially responsible practice.

Bad practice. In the U.K., the future of online gaming is currently being examined. Online gambling legislation will be introduced to permit, regulate, and (most importantly) tax online gaming. This should hopefully attract sites to remain in the U.K. and adhere to legislation as well as a socially responsible practice. Any company that chooses to move to an offshore location where such regulatory control is lax, and void of any socially responsible practice, should obviously be seen with a lower regard. The hope of any legislation is also to attract the customer to well regulated, fair, and socially responsible gaming sites. Any site not displaying these attributes may well lose their customer base.

\section{Built-in pause/confirmation}

When a bet is placed there should be a confirmation message of the details of the bet just placed, with the option to either confirm or decline the bet, giving the customer the chance to change their mind. Where gambling can be rapid and continuous, there should be a built-in pause within games in order to give the customer a chance to reflect on their desire to continue or withdraw.

Good practice. Most sport betting sites do ensure that any bet placed is confirmed by the customer, which gives the customer a chance to rethink as well as reduce mistakes that may be made. The authors would like to see this practice across all online gambling sites.

Bad practice. Whilst the need for pauses is not so relevant for online sports betting, as the activity is quite a slow one, online casinos present a much faster form of gambling. One of the biggest concerns is that online slot machines can be very rapid. No online casino that the authors visited showed any built-in pauses in their play. More worrying was that one online casino had slot machines that spun once a second. This is 3-4 s faster than the industry standard for offline slot machines. Such gambling speed may not only may increase the risk of developing a gambling problem, but can also result in a large sum of money being spent in a very short space of time, without any pauses for the customer to assess their losses or winnings. This issue of pauses in play and confirmation of bets is critical for online casinos as the speed of play on all their 
games are much quicker than those found on sports betting sites. Possible solutions for this could be stopping play on slot machines after every $£ 15$ for example, or every $15 \mathrm{~min}$, so that the customer can reflect on their play. This system should also be put in place for table games and any other game with rapid (re)play.

\section{Practice modes}

It is recognized that many online sites have a practice mode or "demo" format, where a potential customer can place a pretend bet in order to see the procedure of gambling on that site. Although this activity cannot be regarded as actual gambling as there is no "real" money involved, it can be accessed by under-aged gamblers and possibly attract an underage player into gambling. It is therefore recommended that any controlled gambling messages and links to helplines/help-groups that appear on the other pages of the site, should also appear in the practice/"play for fun" section.

Good practice. There are currently no sites with a reference to controlled gambling on their practice modes. The need for such a message is especially relevant for online casinos, where people of any age can download practice modes and play anything from poker to slot machines. Although legally it is not gambling (as there is no money involved), it is questionable whether access to such simulators should be available for young children. It could lead to young people seeing gambling as just a game and disregarding the financial risks involved. If possible, access to such simulators should only be available for those over the legal age, with a clear message of responsible gambling, as well as a reminder that winning virtual money in practice mode does not necessarily mean that you would win when playing for money. The differentiation between the two must be emphasized.

\section{Encouragement to play}

Whilst it is good commercial practice to promote products and provide the customer with information, there should be no encouragement to either reinvest winnings or chase losses. Gamblers should not be enticed to play on.

Good practice. Sites should make it clear and easy for customers to transfer any winnings back into their personal account or credit card. Most sites visited did well on this.
Bad practice. Some sites have been guilty of over emphasizing the option of holding any winnings in their betting account and encouraging further gambling. As well as this, the claims for the time and charges involved for transferring winnings back into a personal account appear to differ wildly. Whilst a lot of sites will transfer money back into a debit or credit card within days at no cost, some other sites claim that such a process may take up to 14 days, whilst other sites charge the customer.

\section{Online gambling: social responsibility checklist}

Finally, based on the findings of this small study, the authors have compiled a list of recommendations to the online gambling industry. The authors are aware that some of the sites examined already practice a level of social responsibility, and have established links with reputable gambling help organisations such as Gamcare. However, most online gambling sites have a lot to gain by being more socially responsible.

From this study, the authors have drawn up a list of guidelines for socially responsible practice. The authors believe that following such guidelines will not only help to protect the underage and those at risk, but will also attract customers, by demonstrating a socially responsible practice. The following list is not exhaustive but represents the ground rules we would see as important regarding an online gambling site. A number of points are equally applicable to terrestrial operations.

- There needs to be a system built into debit and credit card transactions that proves an effective check on age.

- Advertising and promotional material should not target those under 18 years of age, should be honest, fair, and give a balanced message with regard to winning and losing.

- In addition to credit worthiness checks on account holders, limits should be placed on how much they can commit. This can either be a financial limit per session or per day.

- There should be references to the need to keep gambling under control (i.e., a "risks of the game" message) that is a cautionary note rather than a health warning-and these should be sited where they will be read.

- The homepage should carry the logo of the preferred social responsibility partner (e.g., GamCare) and a statement as to why the company is supportive of the partnership. (The latter does 
not have to be on the homepage). This notice should be accompanied with a link to the website of the preferred social responsibility partner.

- A notice to where a gambler who is concerned with their own (or someone else's) gambling can get help should appear at least twice-once prominently mentioned in the text of the site and alongside the menu box on the page where an online customer transfers money into their gambling account.

- Whilst it is good commercial practice to promote products and provide the customer with information, there should be no encouragement to either reinvest winnings or chase losses. Gamblers should not be enticed to play on. The decision must be their own.

- All gambling opportunities (but especially the rapid and interactive games) should have a built-in pause at predetermined intervals, allowing the gambler to reflect on their desire to continue or withdraw. This is particularly important for those who may find it more difficult to stick to self-imposed limits.

- Customers should have the opportunity to selfexclude themselves from an Internet gambling site. A system that enables them to do this should be in place and clearly explained. Any self-exclusion scheme should be easy to carry out and run for at least 6 months.

- Any free practice mode that is offered to the customer must have an appropriate message regarding responsible gambling. We recommend that access to practice modes be denied to those under the legal age to gamble. Giving access to such simulators could encourage someone underage to seek opportunities to gamble for real money.

- Company staff, particularly senior management and customer support personnel, should be trained/made aware of the importance of social impact issues and that there is no conflict between a company policy that maximizes opportunity and minimizes harm.
- The company adopts a culture and practice that is able to demonstrate and believe in a socially responsible approach.

- The site should be attractive enough to encourage online gamblers to use it in preference to those sites that are less well regulated or which are not committed to social responsibility.

- The company commits itself to supporting organizations that provide treatment, training and education for problem gamblers, those at risk, and young gamblers.

- Before launching a new product or developing an existing one, the company consults, commercially in confidence, with the lead body involved with the social impact of gambling.

\section{REFERENCES}

1. Griffiths, M.D. (1999). Gambling technologies: prospects for problem gambling. Journal of Gambling Studies 15:265-283.

2. Griffiths, M.D., \& Wood, R.T.A. (2000). Risk factors in adolescence: the case of gambling, video-game playing and the internet. Journal of Gambling Studies 16:199-225.

3. Griffiths, M.D., \& Parke, J. (2002). The social impact of internet gambling. Social Science Computer Review 20:312-320.

4. National Research Council. (1999). Pathological gambling: a critical review. Washington, DC: National Academy Press.

Address reprint requests to: Professor Mark Griffiths Psychology Division Nottingham Trent University Burton Street Nottingham, NG1 4BU, U.K.

E-mail: mark.griffiths@ntu.ac.uk 
APPENDIX 1: INTERNET GAMBLING SITES STUDIED $(\mathrm{N}=30)$

Company name

Littlewoods

Manny Bernstein Bookmakers

Betachance

Bet-On-Sports

John Smiths Bookmakers Ltd.

Blue Square Limited

City Index

First Stake

Flutter.Com Ltd.

Gamebookers

Goldengoals.ComVentures Inc.

IG Index

Interbet International

Ladbrokes

Netbetsports.Com

Premier5

Simon Bold (Gibraltar) Ltd.

Soccer Betting

Sporting Bet

Sporting Index Ltd.

Sporting Odds Ltd.

Stanley Leisure PLC

Surrey Sports

Tote

UK Betting

Victor Chandler

William Hill
Web address

www.bet247.com

www.bet24hrs.co.uk

www.betachance.com

www.betonsports.co.uk

www.betsmith.com

www.bluesquare.com

www.cityindex.co.uk

www.firststake.com

www.flutter.com

www.gamebookers.com

www.goldengoals.com

www.igindex.com

www.igsports.com

www.inter-bet.com

www.ladbrokes.co.uk

www.ladbrokescasino.com

www.netbetsports.com

www.premier5.com

www.simonbold.com

www.soccer-betting.com

www.sportingbet.com

www.sportingindex.com

www.sportingodds.com

www.stanleybet.co.uk

www.surreysports.com

www.totalbet.com

www.ukbetting.com

www.victorchandler.co.uk www.williamhill.co.uk wWw.williamhillcasino.com 
